\title{
Bio-inspired anisotropic polymeric heart valves exhibiting valve-like mechanical and hemodynamic behavior
}

\author{
Feng Guo ${ }^{1,2 \dagger}$, Chang $\mathrm{Liu}^{3,4 \dagger}$, Rizheng Han ${ }^{1,2}$, Qiang $\mathrm{Lu}^{5}$, Yun Bai ${ }^{1,2}$, Rui Yang ${ }^{1,2}$, Dun Niu ${ }^{4^{*}}$ and \\ Xing Zhang ${ }^{1,2 *}$
}

\begin{abstract}
Native heart valve leaflets with layered fibrous structures show anisotropic characteristics, allowing them to withstand complex mechanical loading for long-term cardiac cycles. Herein, two types of silk fibroin (SF) fiber membranes with anisotropic (ASF) and isotropic (ISF) properties were prepared by electrospinning, and were further combined with poly (ethylene glycol) diacrylate (PEGDA) hydrogels to serve as polymeric heart valve (PHV) substitutes (PEGDA-ASF and PEGDA-ISF). The uniaxial tensile tests showed obvious anisotropy of PEGDA-ASF with elastic moduli of $10.95 \pm 1.09$ and $3.55 \pm 0.32 \mathrm{MPa}$, respectively, along the directions parallel and perpendicular to the fiber alignment, while PEGDA-ISF possessed isotropic property with elastic moduli of 4.54 \pm 0.43 MPa. The PHVs from both PEGDA-ASF and PEGDA-ISF presented appropriate hydrodynamic properties from pulse duplicator tests according to the ISO 5840-3 standard. However, finite element analysis (FEA) revealed the anisotropic PEGDA-ASF valve showed a lower maximum principle stress value (2.20 MPa) in commissures during diastole compared with that from the isotropic PEGDA-ISF valve (2.37 MPa). In the fully open state, the bending area of the PEGDA-ASF valve appeared in the belly portion and near the attachment line like native valves, however, which was close to free edges for the PEGDA-ISF valve. The Gauss curvature analysis also indicated that the anisotropic PEGDA-ASF valve can produce appropriate surface morphology by dynamically adjusting the movement of bending area during the opening process. Hence, anisotropy of PHVs with bio-inspired layered fibrous struc-
\end{abstract}

tures played important roles in mechanical and hydrodynamic behavior mimicking native heart valves.

Keywords: anisotropy, silk fibroin, poly(ethylene glycol), heart valves, finite element analysis

\section{INTRODUCTION}

A variety of biological tissues including blood vessels, skeletal muscles, cartilages, corneas, and heart valves, exhibit anisotropic behavior [1], due to their heterogenic compositions. For example, skeletal muscles contain a large amount of muscle fibers that are comprised of collagen fibril bundles and highly align in one direction, which allows them to withstand load-bearing and directional shock-absorbing [2]. Skeletal muscles with hierarchical structures also provide appropriate mobility to ensure a number of human body postures, joint movements and other intrinsic physiological functions [3]. Another example is the heart valve, in which aortic valve leaflets with layered structures (fibrosa, spongiasa and ventricularis) exhibit typical anisotropic characteristics. Circumferentially aligned type I collagen fibers mainly in the fibrosa layer bear tensile loads during valve opening [4], while the elastin fibers radially oriented in the ventricularis layer aid in leaflet recoil during valve closing $[5,6]$. Consequently, heart valves are stiffer in the circumferential direction and more deformable in the radial direction (anisotropy), which play an important role in

\footnotetext{
${ }^{1}$ Institute of Metal Research, Chinese Academy of Sciences, Shenyang 110016, China

${ }^{2}$ School of Materials Science and Engineering, University of Science and Technology of China, Hefei 230026, China

${ }^{3}$ Institute of Textiles and Clothing, The Hong Kong Polytechnic University, Hong Kong, China

${ }^{4}$ Department of Chemistry, Northeastern University, Shenyang 110004, China

${ }^{5}$ National Engineering Laboratory for Modern Silk \& Collaborative Innovative Center of Suzhou Nano Science and Technology, Soochow University, Suzhou 215123, China

$\dagger$ These authors contributed equally to this work.

* Corresponding authors (emails: xingzhang@imr.ac.cn (Zhang X); niudun666@hotmail.com (Niu D))
} 
achieving large effective orifice area (EOA), low pressure gradient and particularly steady blood flow during the cardiac cycle. Hence, anisotropy of heart valves not only is critical to their mechanical properties, but also influences hemodynamic functions.

Patients with severe valve disease need prosthesis replacement by mechanical heart valves (MHVs) or bioprosthetic heart valves (BHVs). Mechanical properties of inflexible MHVs differ greatly from human valves, which would lead to hemodynamic issues. BHVs possess proper mechanical characteristic, but are prone to slow calcification and enzymatic hydrolysis, leading to limited lifetime. Polymeric heart valves (PHVs) have been proposed as promising candidates to overcome the drawbacks of the BHVs with better durability and enhanced hydrodynamic functions [7]. More importantly, polymeric composites can be developed with specific micro-structures to mimic the anisotropy and layered structures of native heart valves. For example, Tseng et al. [8] fabricated composites from poly(ethylene glycol) (PEG) hydrogels and poly(caprolactone) (PCL) fibers with mechanical anisotropy as an analog to the fibrosa. Moreover, a tri-layered scaffold fabricated from microfabricated poly(glycerol sebacate) (PGS) and fibrous PGS/ PCL exhibited tunable mechanical anisotropy similar to native heart valves [9]. Polyglycolic acid (PGA) scaffolds with microfiber alignment on a centimeter scale were prepared by the stretching technique, which showed a high level of anisotropy [10]. However, these studies mainly focused on the mechanical properties of the anisotropic polymeric scaffolds. The effects of anisotropy on hydrodynamic properties and stress-strain distribution of PHVs during cardiac cycles still remain poorly understood.

Finite element analysis (FEA) can provide quantitative stress and strain distribution on heart valve leaflets at the microscopic level during the opening and closing cycles. Anisotropic constitutive models of heart valves including orthotropic bovine pericardium [11], transverse fiber mode [12] and three-dimensional (3D) anisotropic hyperelasticity [13] have been developed. FEA based on these models indicated that anisotropy played important roles in stress relaxation of heart valve leaflets during the cardiac cycles $[14,15]$. These finite element models were primarily applied to biological tissues (such as porcine valves and bovine pericardium), but not yet for anisotropic PHVs.

In this study, isotropic and anisotropic silk fibroin (SF) membranes were produced by electrospinning methods. SF membranes were further used to fabricate PHVs to- gether with poly(ethylene glycol) diacrylate (PEGDA) hydrogels, which exhibited excellent biocompatibility and low-fouling property [16]. The microstructures, mechanical properties and hemodynamic performance of these PHVs were studied. The influence of anisotropy on the stress-strain distribution and hemodynamic behavior of these PHVs was investigated by FEA.

\section{EXPERIMENTS AND METHODS}

\section{Electrospinning of SF membranes}

SF was prepared following a previously reported method with minor modification [17]. Raw silk (Ni's Silk Spinning Factory, Suzhou, Jiangsu, China) was boiled in a $0.02 \mathrm{~mol} \mathrm{~L}^{-1} \mathrm{Na}_{2} \mathrm{CO}_{3}$ solution for $30 \mathrm{~min}$ and then rinsed thoroughly with ultrapure water (A 10, Millipore, Billerica, MA, USA) to remove sericin proteins. The silk sample was further dissolved in a $9.3 \mathrm{~mol} \mathrm{~L}^{-1} \mathrm{LiBr}$ solution at $60^{\circ} \mathrm{C}$ and dialyzed against ultrapure water using a dialysis tube (molecular weight (M.W.) cut-off 8000 14,000) for $48 \mathrm{~h}$. The solution inside the dialysis tube was then concentrated with PEG (M.W. $=20,000 \mathrm{~g} \mathrm{~mol}^{-1}$, Wokai Biotechnology Co., Ltd., Beijing, China). Different concentrations of SF solutions (10 wt.\%, 13 wt.\% and 20 wt.\%) were tried for electrospinning of SF mats. A concentration of 13 wt.\% SF solution was chosen for electrospinning of the samples in the following experiments.

The viscosity was adjusted by adding $5 \mathrm{~mL}$ of $5.0 \mathrm{wt} . \%$ poly(ethylene oxide) (PEO, M.W.=300,000 $\mathrm{g} \mathrm{mol}^{-1}$ ) into $10 \mathrm{~mL}$ of $13 \mathrm{wt} . \% \mathrm{SF}$ solution before electrospinning. The rotating cylindrical receiver of electrospinning machine (Ucalery Ltd., Beijing, China) was used to collect directional SF fibers, and the baffle receiver was used to collect randomly aligned SF fibers. The feeding rate was $0.8 \mathrm{~mL}$ per hour. The distance between the tip and the collector $(10,15$ and $20 \mathrm{~cm})$ and voltage $(18,21$ and $23 \mathrm{kV})$ were adjusted in order to obtain long and uniform SF fibers by electrospinning. The distance between the tip and the collector was set as $15 \mathrm{~cm}$, while a positive voltage $(13 \mathrm{kV})$ and a negative voltage of $-8 \mathrm{kV}$ were applied for electrospinning of the samples in the following experiments.

In this study, three types of SF membranes were fabricated to obtain isotropic and anisotropic materials. The randomly aligned SF (isotropic SF, hereafter named as ISF) membrane and directional aligned SF (hereafter named as DSF) membrane were prepared using the baffle receiver and rotating cylindrical receiver (rotation speed of $2800 \mathrm{r} / \mathrm{min}$ ) for $12 \mathrm{~h}$, respectively. An anisotropic SF (hereafter named as ASF) membrane combining DSF and 
ISF was fabricated through collection first with rotating cylindrical receiver (rotation speed of $2800 \mathrm{r} / \mathrm{min}$ ) for $7 \mathrm{~h}$, followed by a baffle receiver for $5 \mathrm{~h}$. All membrane samples were immersed into $90 \%(v / v)$ methanol solutions (in water) at room temperature for $30 \mathrm{~min}$, and then thoroughly rinsed with distilled water for $48 \mathrm{~h}$ to remove PEO [18].

\section{PEGDA synthesis}

PEGDA was synthesized following a previously described method with minor modification [19]. PEG powder (26.8 g, M.W.=3.35 kDa, Sigma-Aldrich, St. Louis, MO, USA) was dissolved in $80 \mathrm{~mL}$ anhydrous dichloromethane (DCM, J\&K Scientific Ltd., Beijing, China) in a round-bottom flask $(500 \mathrm{~mL})$, and then $2.24 \mathrm{~mL}$ triethylamine (TEA, Sigma-Aldrich) and $2.54 \mathrm{~mL}$ acryloyl chloride (Tokyo Chemical Industry, Tokyo, Japan) were subsequently dropped into the PEG solution. The whole reaction was carried out in argon atmosphere and the resulting PEGDA was purified and lyophilized, and finally stored at $-20^{\circ} \mathrm{C}$ for further use. The proton nuclear magnetic resonance $\left({ }^{1} \mathrm{H}\right.$ NMR) spectrum of the asobtained PEGDA was performed using a Bruker $400 \mathrm{MHz}$ spectrometer (AVANCE II 400, Bruker, Karlsruhe, Germany) to verify the percentage of acylation (Fig. S1).

\section{Preparation of PEGDA-ISF and PEGDA-ASF composites}

PEGDA powder was dissolved in a phosphate buffered solution (PBS) to obtain a $20 \mathrm{wt} . \%$ PEGDA solution. The UV initiator Irgacure 2959 (Tokyo Chemical Industry, Tokyo, Japan) was first dissolved in ethanol, which was further diluted in the above PEGDA solution to obtain a concentration of $3.0 \mathrm{~g} \mathrm{~L}^{-1}$. The ISF and ASF membranes punched with an array of holes (Fig. 1a) using a stainlesssteel needle were then immersed into the PEGDA solution under vacuum for $30 \mathrm{~min}$ to remove air bubbles. These samples were cut into stripes $\sim 78.5 \mathrm{~mm} \times 30 \mathrm{~mm}$, which were then transferred into polydimethylsiloxane (PDMS) molds ( $78.5 \mathrm{~mm} \times 30 \mathrm{~mm} \times 400 \mu \mathrm{m})$ and sealed by two pieces of glass slides pretreated with sigmacote (Sigma-Aldrich, St. Louis, MO, USA). These samples were further exposed to UV light (B-100 AP, UVP company, Upland, CA, USA) for 7 min to obtain PEGDA-ISF and PEGDA-ASF composites (thickness $\sim 400 \mu \mathrm{m}$ ), respectively. Staining of PEGDA hydrogels using a 0.5 wt.\% crystal violet solution (Dingguo Changsheng biotechnology Co., Ltd, Beijing, China) was performed to confirm that the holes were filled with

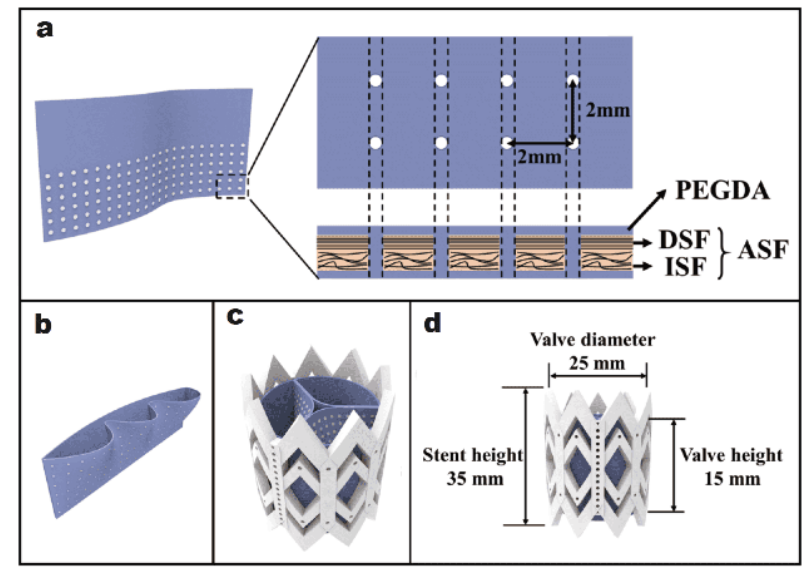

Figure 1 Schematic drawing for fabrication of a PHV from the PEGDA-ASF composite. (a) The top view and cross section of the PEGDAASF composite with layered fibrous structures, (b) assembly of PEGDAASF leaflets, (c) assembly of the PEGDA-ASF valve on the stent, and (d) diameter and height sizes for the valve and stent.

PEGDA hydrogels.

\section{Morphology and chemical composition analyses}

In order to investigate the morphology and chemical composition of SF membranes, ISF, DSF, ASF, PEGDAISF, and PEGDA-ASF samples were dehydrated in a series of ethanol-water solutions with different ethanol concentrations $(50 \%, 70 \%, 90 \%, 100 \%)$ for $15 \mathrm{~min}$ each time. These samples were mounted on an aluminum plate with conductive adhesive and morphologies were observed using a field emission scanning electron microscope (SEM, Supra 35, Zeiss, Oberkochen, Germany) at an accelerating voltage of $20 \mathrm{kV}$. Moreover, attenuated total reflection-Fourier transform infrared (ATR-FTIR) spectra with the wavenumber range of $600-4000 \mathrm{~cm}^{-1}$ were obtained to investigate the conformational transition of SF using an FTIR spectrometer (Tensor27, Bruker, Karlsruhe, Germany). Calorimetric measurement was conducted in the temperature range of $25-100^{\circ} \mathrm{C}$ at a rate of $10^{\circ} \mathrm{C} / \mathrm{min}$ using TGA/DSC 1 instrument (Mettler Toledo, Greifensee, Switzerland).

\section{Mechanical tests}

All samples were cut into $20 \mathrm{~mm} \times 4 \mathrm{~mm}$ stripes for uniaxial tensile tests in water at room temperature using a TA Electroforce ELF 3200 system (Eden Prairie, MN, USA), with the crosshead speed of $0.02 \mathrm{~mm} \mathrm{~s}^{-1}$ and the gauge length of $10 \mathrm{~mm}$. In particular, PEGDA-ASF samples were cut into stripes along different directions towards the fiber alignment direction (parallel $\theta=0^{\circ}$, perpendicular $\theta=90^{\circ}$, and $\theta=45^{\circ}$ ) (Fig. S2a). Five re- 
plicates for each sample were tested. The loading force and displacement in each test were recorded, and divided by the cross-sectional area and gauge length of the samples to obtain the stress and strain values, respectively. The elastic modulus of each sample was determined by the slope of the linear region (in the strain range of 0 $0.15)$ in the stress-strain curve. The in-plane shear moduli of these PEGDA-ASF samples were calculated using the $45^{\circ}$ off-axis tension method as previously described [20]. Moreover, the shape change of a markline in the middle position of the sample through the tensile test was recorded by a CCD camera (Nikon D5200, Tokyo, Japan) (Fig. S2b). Poisson's ratio of the sample was calculated by the negative ratio between the length strain and axial strain for the markline based on the video record.

\section{Preparation of PHVs}

Heart valve stents with cylindrical mesh structures of $\sim 25 \mathrm{~mm}$ in diameter and $\sim 35 \mathrm{~mm}$ in height were fabricated and used to assemble artificial leaflets together as PHVs (transcatheter mode). These stents were fabricated by Form 2 desktop SLA 3D printer (Formlabs, Somerville, MA, USA) using FLGPGRO2 resin. PEGDA-ISF and PEGDA-ASF composites in rectangular shapes $(78.5 \mathrm{~mm}$ $\times 30 \mathrm{~mm}$ ) were folded as artificial leaflets and sutured to form three identical pockets as shown in Fig. 1a, b. These artificial leaflets were further fixed into the stent, ensuring SF fibers aligning to the circumferential direction similar to native valve leaflets (Fig. 1c, d).

\section{Pulse duplicator tests}

In vitro hemodynamic properties of PHVs were tested in a $0.9 \%$ saline solution using a pulse duplicator system (Vivitro Labs, Inc., British Columbia, Canada) according to the ISO 5840-3 Standard [21]. EOA and regurgitant fraction $(\mathrm{RF})$ were calculated based on the data recorded for 10 cycles using the ViViTest software (Vivitro Labs, Inc., British Columbia, Canada), following the equations as below.

$$
Q_{\mathrm{rms}}=\sqrt{\frac{\int_{t_{2}}^{t_{1}} Q(t)^{2} \mathrm{~d} t}{t_{2}-t_{1}}},
$$

where $Q(t)$ is the instantaneous flux, while $t_{1}$ and $t_{2}$ are the starting time and ending time of the forward flow, respectively.

$$
\mathrm{EOA}=\frac{Q_{\mathrm{rms}}}{51.6 \sqrt{\frac{\Delta P}{\rho}}},
$$

where $\Delta P(\mathrm{mmHg}, 1 \mathrm{mmHg} \approx 133.32 \mathrm{~Pa})$ is the mean pressure difference, and $\rho\left(\mathrm{g} \mathrm{cm}^{-3}\right)$ is the density of the test fluid.

$\mathrm{RF}=\frac{\int_{t_{4}}^{t_{3}} Q(t) \mathrm{d} t}{\mathrm{SV}} \times 100 \%$,

where SV $\left(\mathrm{L} \mathrm{min}^{-1}\right)$ is the stroke volume of cardiac output, and $t_{3}$ and $t_{4}$ are the starting time and ending time of the backward flow, respectively.

\section{FEA for mechanical properties}

FEA was performed to investigate the mechanical properties of PHVs using PEGDA-ASF and PEGDA-ISF composites. For simplification, a nearly linear relationship between the stress and strain for these composites in a small deformation range was hypothesized. Hence, the anisotropic form of Hooke's law can be expressed in Voigt notation as below:

$\sigma_{i}=C_{i j} \varepsilon_{j}$,

where $\sigma_{i}$ is the true stress, $\varepsilon_{i}$ is the true strain and $C_{i j}$ is the stiffness matrix of the material. Taking the unidirectional feature of SF fibers in the PEGDA hydrogel matrix into account, the PEGDA-ASF composite can be simply considered as a transversely isotropic material, thus, the stiffness matrix would be expressed as:

$C_{i j}=\left[\begin{array}{lllllll}C_{11} & C_{12} & C_{13} & & & \\ C_{21} & C_{22} & C_{23} & & & \\ C_{12} & C_{23} & C_{22} & & & \\ & & & \frac{C_{22}-C_{23}}{2} & & \\ & & & & C_{66} & \\ & & & & & C_{66}\end{array}\right]$,

where

$C_{11}=\frac{1-v_{23} v_{32}}{E_{2}^{2} \Delta}$,

$C_{12}=\frac{v_{12}+v_{12} v_{32}}{E_{1} E_{2} \Delta}$

$C_{22}=\frac{1-v_{13} v_{31}}{E_{1} E_{2} \Delta}$,

$C_{23}=\frac{v_{23}-v_{21} v_{13}}{E_{1} E_{2} \Delta}, C_{66}=G_{12}$,

$\Delta=\frac{1}{E_{1} E_{2}^{2}}\left|\begin{array}{ccc}1 & -v_{21} & -v_{31} \\ -v_{12} & 1 & -v_{32} \\ -v_{13} & -v_{23} & 1\end{array}\right|$,

$\frac{v_{i j}}{E_{i}}=\frac{v_{j i}}{E_{j}}$.

Young's modulus $E_{i}$ and shear modulus $G_{12}$ were obtained from the uniaxial tensile tests. Poisson's ratio $v_{12}$ was calculated based on images from the deformation 
process, and $v_{23}=v_{\mathrm{PEGDA}}$.

Meanwhile, the randomly aligned PEGDA-ISF composites can be considered as a simple linear isotropic incompressible material, which showed a Young modulus of $8 \mathrm{MPa}$ and Poisson's ratio of 0.45 . Moreover, leaflet prototypes with the geometry of $25 \mathrm{~mm}$ in diameter and $15 \mathrm{~mm}$ in height were built. The Abaqus/Explicit solver (v6.13, Simulia, Dassault Systems, Providence, RI, USA) was used to carry out the simulation analysis owing to highly nonlinear characteristics of leaflets due to large deformation and convergence issue. The valve model was imported into Abaqus/Explicit solver to obtain meshed 8000 S4 shell elements with a thickness of $0.4 \mathrm{~mm}$. Density of the leaflets was assumed to be $1,100 \mathrm{~kg} \mathrm{~m}^{-3}$ and the frame was considered to be rigid and fixed. Furthermore, the transvalvular pressure waveform obtained from in vitro pulse duplicator tests was applied to simulate the dynamic loading for the leaflets.

The coordinate points on the leaflet surfaces in the fully open state were extracted and imported into Matlab 2012b (Mathworks Inc., Natick, MA, USA) to calculate curvature distribution of the leaflet surface. The Gauss curvature $\left(K^{\text {Gauss }}\right)$ that is defined by the product of principal curvatures has been employed.

$$
K^{\text {Gauss }}=\kappa_{1} \kappa_{2} \text {, }
$$

where $\kappa_{1}$ is the maximum value, and $\kappa_{2}$ is the minimum value. Moreover, Gauss curvature can be expressed by fundamental coefficients of the surface.

$$
K^{\text {Gauss }}=\frac{L N-M^{2}}{E G-F^{2}},
$$

where $E, F$ and $G$ are the first fundamental coefficients of the surface, and $L, M$ and $N$ are the second fundamental coefficients of the surface. A mesh surface was first fitted by the coordinates of the leaflet, and the discrete points on the mesh were then calculated to obtain all parameter values in Equation (10).

\section{Statistical analysis}

All data were expressed as mean \pm standard deviation (S.D.). Statistical significance was analyzed by one-way analysis of variance (ANOVA) with post hoc Tukey testing. The $p$ value less than 0.05 was considered to be statistically significant.

\section{RESULTS}

\section{Morphology and chemical composition of samples}

The average thickness of DSF, ISF and ASF membranes was $320 \mu \mathrm{m}$. ASF membranes were prepared by com- bining a DSF membrane (thickness $\sim 200 \mu \mathrm{m}$ ) with an ISF membrane (thickness $\sim 120 \mu \mathrm{m}$ ). Fig. $2 \mathrm{a}$ shows that fibers of the DSF membrane predominantly align in one direction (Fig. 2a), while fibers in the ISF membrane randomly orient (Fig. 2b). The fibers' morphologies of the ASF membrane from both DSF and ISF layers are shown in Fig. 2c. The two layers were tightly bound together after electrospinning based on the cross-section view (Fig. 2d). A thin layer of PEGDA hydrogels formed on both top and bottom surfaces of the ASF membrane in the PEGDA-ASF samples. SEM images of PEGDA-ASF composites (Fig. 2e, f) show the uniform thin layer of PEGDA hydrogels (thickness $\sim 20 \mu \mathrm{m}$ after dehydration) atop of the ASF membrane. The cross section images (Fig. 2f, g) show the tight bonding between the ASF membrane and PEGDA hydrogels. Crystal violet staining indicated that punched holes in the PEGDA-ASF composites were filled with PEGDA hydrogels (Fig. S3a, b), confirming the penetration of PEGDA hydrogels through these hole arrays.

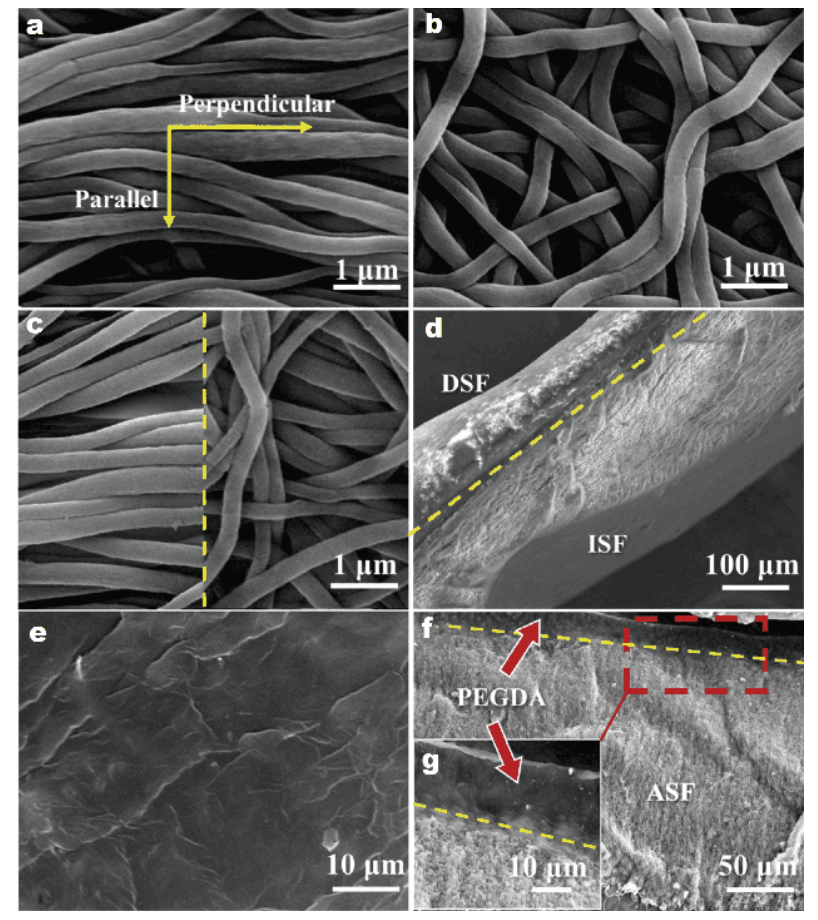

Figure 2 SEM images of fiber morphologies of (a) a DSF membrane, (b) an ISF membrane, (c) top (left) and bottom (right) layers of an ASF membrane, and (d) the side view of an ASF membrane with layered structures and tight bonding between DSF and ISF membranes. SEM images of (e) surface morphology and (f) cross section of a PEGDA-ASF composite. The enlarged image $(\mathrm{g})$ of the red dotted rectangular area shows a uniform thin layer of PEGDA hydrogel atop of the ASF membrane and the yellow dashed line in $(\mathrm{g})$ represents the boundary between the ASF membrane and PEGDA hydrogel. 
Structural changes for the SF membrane after methanol treatment are demonstrated by the ATR-FTIR spectra as shown in Fig. 3a. The original SF membrane showed the characteristic vibration band at $1651 \mathrm{~cm}^{-1}$ (amide I) corresponding to the random-coil/a-helix, while bands at $1544 \mathrm{~cm}^{-1}$ (amide II), $1537 \mathrm{~cm}^{-1}$ (amide II) and $1240 \mathrm{~cm}^{-1}$ (amide III) were attributed to the random-coil $[22,23]$. After treatment with methanol, the SF membrane exhibited characteristic bands of the $\beta$-sheet at $1620 \mathrm{~cm}^{-1}$ (amide I), $1510 \mathrm{~cm}^{-1}$ (amide II) and $1230 \mathrm{~cm}^{-1}$ (amide III), indicating the conformation converted from random-coil/ $\alpha$-helix to $\beta$-sheet [22]. The random-coil/ $\alpha$-helix structure is generally water-soluble, while $\beta$-sheet is insoluble. Therefore, conformational transition from the random-coil/a-helix structure to $\beta$-sheet by methanol treatment can stabilize SF fibers in the aqueous condition. An endothermic peak around $62.5^{\circ} \mathrm{C}$ was present in the differential scanning calorimetry (DSC) curve for the original SF sample (Fig. 3b), likely attributed to the melting of PEO [24]. On the other hand, there was no endothermic peak around $62.5^{\circ} \mathrm{C}$ for the SF membrane after PEO extraction, indicating $\mathrm{PEO}$ components in the fibrous scaffolds were completely removed (Fig. 3b).

\section{Mechanical properties of samples}

The representative stress-strain curves of ISF and ASF membranes are shown in Fig. 4a, which consist of linear (strain $\sim 0-0.15$ ) and non-linear (strain $>0.15$ ) regimes. The elastic moduli (the linear regime) of ISF membranes are $6.34 \pm 0.43 \mathrm{MPa}$, whereas the elastic moduli are 12.11 \pm 0.61 and $3.44 \pm 0.29 \mathrm{MPa}$ along the directions of $\theta=0^{\circ}$ and $\theta=90^{\circ}$, respectively, for ASF membranes (Fig. 4b). Therefore, ASF membranes show mechanical anisotropy with higher elastic moduli of ASF in the direction parallel to the fiber alignment $\left(\theta=0^{\circ}\right)$ than that along the direction perpendicular to the fiber alignment $\left(\theta=90^{\circ}\right)$. The representative stress-strain curves of PEGDA-ASF and PEGDA-ISF composites are shown in Fig. 4c, which also consist of linear (strain $\sim 0-0.15$ ) and non-linear (strain $>0.15$ ) regimes. Although the PEGDA hydrogels are isotropic with low elastic moduli $(0.56 \pm 0.03 \mathrm{MPa}$, Fig. S4), PEGDA-ASF composites show mechanical anisotropy characteristic. The elastic moduli (the linear regime) of PEGDA-ASF composites with a thickness of $400 \mu \mathrm{m}$ are $10.95 \pm 1.09$ and $3.55 \pm 0.32 \mathrm{MPa}$ along the directions of $\theta=0^{\circ}$ and $\theta=90^{\circ}$, respectively, close to those of native heart valves as previously reported (Table S1) [25-27]. On the other hand, PEGDA-ISF composites present isotropic characteristic with elastic moduli of $4.54 \pm 0.43 \mathrm{MPa}$ (Fig. 4d).

\section{Hemodynamic properties of PHVs}

Fig. 5a shows the morphology of the PHV from PEGDAASF composites in the opening and closing cycles and corresponding hydrodynamic performance in vitro. The hydrodynamic curve of the PHV (Fig. 5b) exhibits hydrodynamic performance close to native valve with a low pressure difference (Table S1) and large EOA value in the systole. The large EOA value $\left(2.6 \mathrm{~cm}^{2}\right)$ for the PHV indicates low energy loss [28]. The RF is another key factor that indicates the summation of retrograde blood during the closing motion of the prosthesis and leakage flow after closure. The blue curve marked in Fig. 5b represents flow waveform in one cardiac cycle, which is divided into three parts: the forward flow (f1-f2), closing volume (f2-f3), and leakage volume ( $\mathrm{f} 3-\mathrm{f} 4)$. The relatively large $\mathrm{RF}$ value (20\%) for the PHV from PEGDA-ASF composites may be due to the leakage after valve closure. PEGDA-ISF pre-
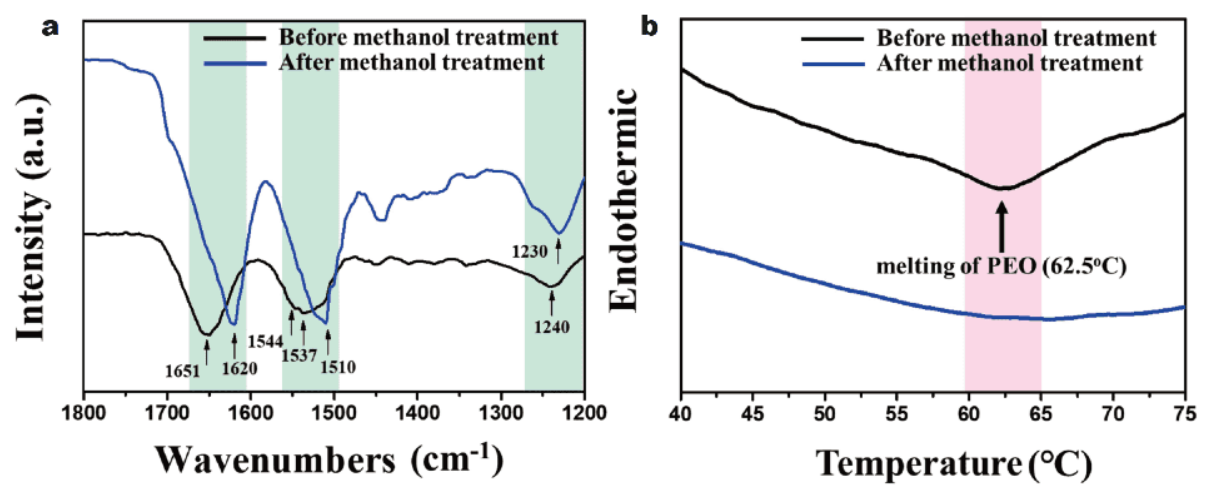

Figure 3 (a) ATR-FTIR spectra of an original SF membrane (black) and an SF membrane after methanol treatment (blue). Characteristic bands shown in the blue shadow confirm the conversion from random-coil/a-helix to $\beta$-sheet of SF after methanol treatment. (b) DSC curves of the SF membrane before (black) and after (blue) methanol treatment confirm the disappearance of the endothermic peak (the red shadow) for PEO melting after methanol treatment. 

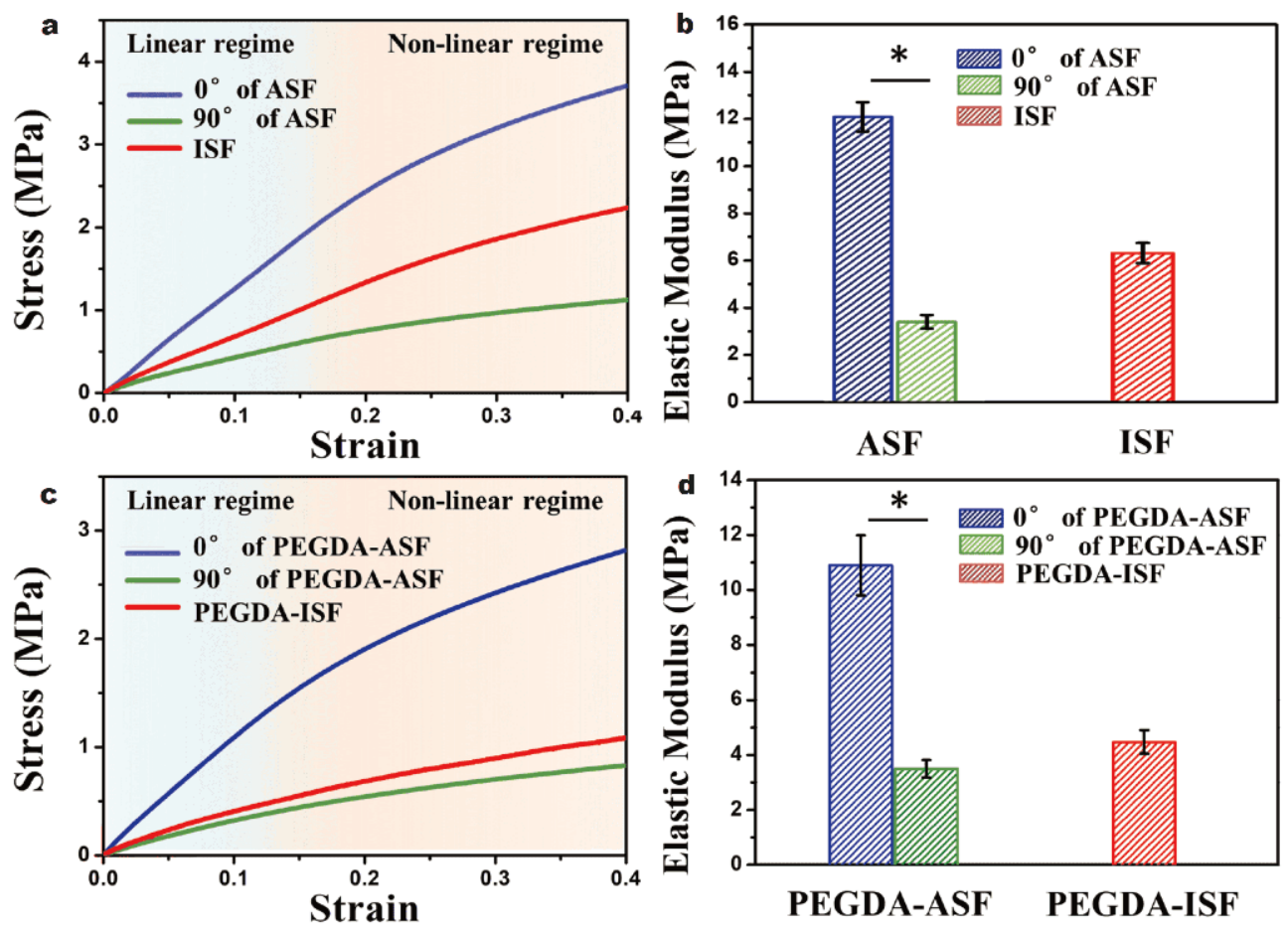

Figure 4 (a) Representative stress-strain curves of ISF and ASF samples along the directions of $\theta=0^{\circ}$ and $\theta=90^{\circ}$, and (b) elastic moduli of ISF and ASF samples. (c) Representative stress-strain curves of PEGDA-ISF and PEGDA-ASF samples along the directions of $\theta=0^{\circ}$ and $\theta=90^{\circ}$, and (d) elastic moduli of PEGDA-ASF and PEGDA-ISF samples. ${ }^{*} p<0.001$ indicates significant difference among groups.

sents similar hydrodynamic property with an EOA value of $2.3 \mathrm{~cm}^{2}$ and an RF value of $14.5 \%$ (Fig. $5 \mathrm{c}$ and Fig. S5). Moreover, the EOA and RF values for both PHVs from PEGDA-ASF and PEGDA-ISF meet the requirements defined by ISO 5840-3 Standard [21], in which the EOA value should be equal to or larger than $1.45 \mathrm{~cm}^{2}$ and the $\mathrm{RF}$ value should be equal to or less than $20 \%$ for a transcatheter valve with diameter of $25 \mathrm{~mm}$.

\section{FEA for mechanical behavior of PHVs}

Engineering stress and strain generally fail to reflect the real stress and strain of materials at large deformation. Hence, the true stress-strain curves of PEGDA-ISF and PEGDA-ASF samples considered to be incompressible were adopted along three directions $\left(\theta=0^{\circ}, \theta=90^{\circ}\right.$ and $\theta=45^{\circ}$ ) from uniaxial tensile tests (Fig. 6a) [29]. The true stress-strain curves showed linear relationships, and Young's moduli $E_{1}$ and $E_{2}$ were further attained from the slope of the linear-fitting curves along the directions of $\theta=0^{\circ}$ and $\theta=90^{\circ}$, respectively, for PEGDA-ASF samples. Shear modulus $G_{12}$ of the PEGDA-ASF sample was calculated by the Young's modulus along the direction of $\theta=45^{\circ}$ [20]. In addition, vertical and axial strains of the samples were calculated based on the displacement of the markline during the tensile test, which also exhibited a linear relationship (Fig. 6b). Poisson's ratios, $v, v_{12}$ and $v_{23}$ were calculated using ratios of vertical strain/axial strain for PEGDA-ISF, PEGDA-ASF $\left(\theta=0^{\circ}\right)$ and PEGDA hydrogel samples, respectively. Parameters for mechanical properties of PEGDA-ASF and PEGDA-ISF samples were then obtained as shown in Table 1. Local material orientation for the PEGDA-ASF leaflet at each element was defined as shown in Fig. 6c. Material orientations 1, 2 and 3 were along the circumferential direction, radial direction and perpendicular to the leaflet surface, respectively, consistent with the subscript characters in the Table 1. Moreover, the diagram of subareas of valve leaflet was shown in Fig. 6d [30] for further illustration of the simulation results.

The contour plots and vector plots of the maximum inplane principal true strain at fully open and closed state for the PEGDA-ISF and PEGDA-ASF leaflets are shown in Fig. 7. In the fully open state, positive peak strain (red color) was observed near the commissure region, whereas negative peak strain (dark blue color) was shown in the bending area for both PEGDA-ISF and PEGDA-ASF leaflets (Fig. $7 \mathrm{a}, \mathrm{b}$ ). Vector plots also proved that the deformation direction of bending area was obviously 

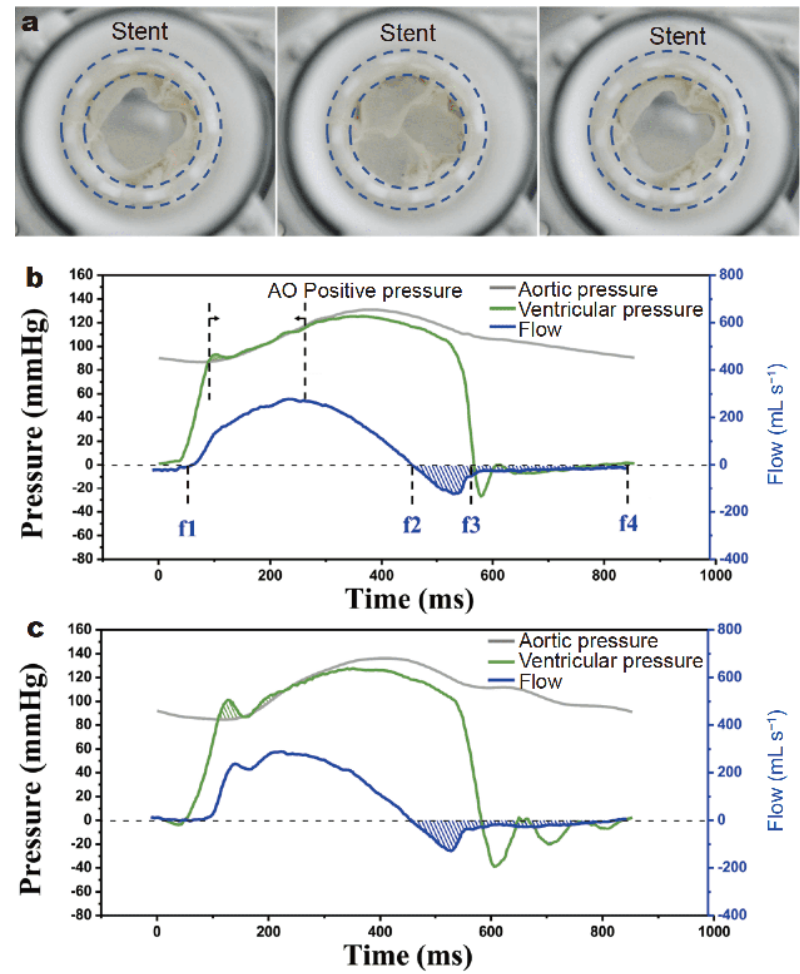

Figure 5 (a) Morphologies of the PHV from PEGDA-ASF composites in the open and closed state under hemodynamic tests using a pulse duplicator. Pressure and flow rate waveforms in a cardiac cycle for the PHVs from (b) PEGDA-ASF and (c) PEGDA-ISF composites. Green shadow areas in $(b, c)$ indicate low pressure differences during systole, and blue shadow areas are used for calculation of the total regurgitant fraction during diastole.

distinct from other parts of the leaflet. The bending area of the PEGDA-ISF leaflet was close to free edge (marked by blue dotted line), however, which appeared in belly near attachment line for the PEGDA-ASF leaflet (marked by red dotted line). In the fully closed state, large strain concentrated in the commissure along the circumferential direction was found for both PEGDA-ISF and PEGDAASF leaflets (Fig. 7c, d). The peak strain value (0.478) for the PEGDA-ISF leaflet was much higher than that for the PEGDA-ASF leaflet (0.224).

The maximum principal stress distributions of the PEGDA-ISF valve and PEGDA-ASF valve during a cardiac cycle are depicted in Fig. 8. In the fully open configuration, the positive peak stress (red color) for the PEGDA-ASF leaflet was $0.925 \mathrm{MPa}$ in the junction of free edge and commissure, larger than that for the PEGDAISF leaflet $(0.55 \mathrm{MPa})$, probably due to higher elastic modulus of the former compared with the latter. The negative peak stress (dark blue color) concentrated in the bending area for both models (Fig. 8a, b). The stress vector plots proved that the positive stress was caused by stretching when leaflets were open, whereas negative stress was related to bending (Fig. S6). The obtained peak values at the commissure for the PEGDA-ISF and PEGDA-ASF leaflets in the fully closed state were 2.37 and 2.20 MPa, respectively, and stress distribution in the latter was more uniform (Fig. 8c, d). Moreover, the positive stresses of all elements in the fully open state of the valve leaflet were extracted, and the interval frequencies were counted according to the relative proportion of the maximum values (Fig. 8e). There was a higher proportion of large stress for the PEGDA-ISF leaflets compared with the PEGDA-ASF leaflets (the blue dotted square), indicating more severe stress concentration for the PEGDA-ISF valve in the closed state.

Simulation results also showed obvious morphology difference from PEGDA-ISF and PEGDA-ASF valves during cardiac cycle. Hence, the coordinate points on the leaflet surfaces were extracted to evaluate the curvature change in the fully open state. The change of Gauss curvature along the central line of the valve leaflet with distance at different time points was calculated (Fig. 9a). The wave of curvature derivative corresponded to the bending portion with large deformation in the PEGDAISF and PEGDA-ASF leaflets. Bending areas of the PEGDA-ISF leaflets retained in the belly during the opening process. However, there was obvious movement of bending areas for the PEGDA-ASF leaflets during the opening process. At the beginning $(t=0 \mathrm{~ms})$, the PEGDAASF leaflets were partially open and produced a bending area in the belly (marked by blue dotted line). At $t=8 \mathrm{~ms}$, the bending area moved from the belly to free edge with even higher amplitude of curvature derivative. Further deformation of the bending area in the free edge for the PEGDA-ASF leaflets was observed at $t=20 \mathrm{~ms}$, meanwhile another bending area (marked by red dotted line) was shown close to the attachment line. In the fully opened state $(t=40 \mathrm{~ms})$, only the bending area close to the attachment line (marked by black dotted line) was present. The contour plots of Gauss curvature (Fig. 9b, c) showed that peak values for the PEGDA-ISF leaflet found in commissure and center of bending area were much higher than those from the PEGDA-ASF leaflet in the fully open state $(t=40 \mathrm{~ms})$.

\section{DISCUSSION}

Native heart valve leaflets show complex 3D architectures comprising macroscopically inter-connected layers and microscopically fibrous structures that give them anisotropic characteristics to fulfill mechanical and hemody- 

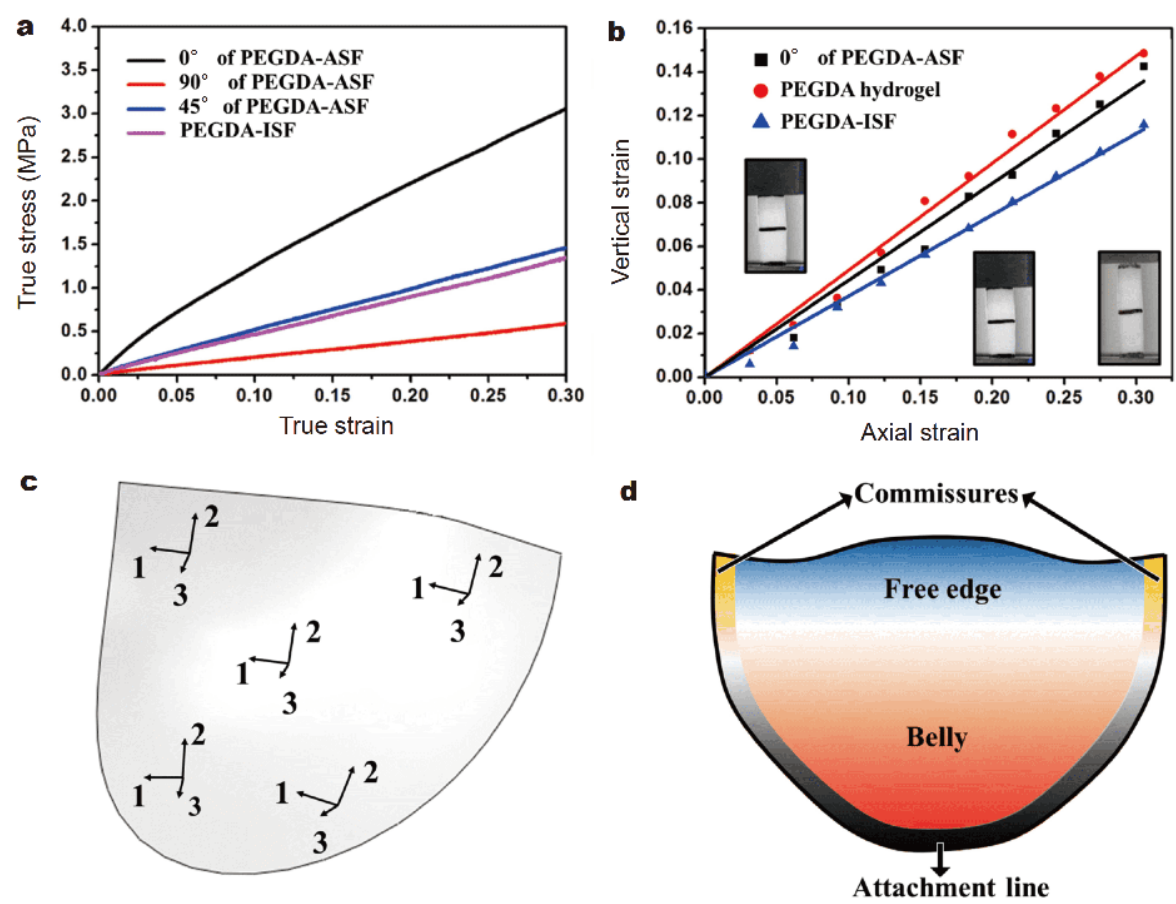

Figure 6 (a) Representative true stress-strain curves for PEGDA-ASF samples along different directions $\left(\theta=0^{\circ}, 90^{\circ}\right.$ and $\left.45^{\circ}\right)$ and PEGDA-ISF sample. (b) The fitted linear relationships between axial strain and vertical strain for PEGDA-ASF samples in $\theta=0^{\circ}$ direction, PEGDA hydrogel samples and PEGDA-ISF samples in uniaxial tensile tests, and the slope of the fitting line represents the Poisson's ratio of the sample. (c) Schematic diagram of material orientation in the PEGDA-ASF leaflet. (d) Subarea names of a valve leaflet: commissures (yellow shadow), free edge (blue shadow), belly (red shadow) and attachment line (black shadow) [30].

Table 1 Parameters for FEA of mechanical properties of PEGDAASF and PEGDA-ISF samples

\begin{tabular}{cccccc}
\hline \multirow{2}{*}{ PEGDA-ASF } & $E_{1}(\mathrm{MPa})$ & $E_{2}(\mathrm{MPa})$ & $v_{12}$ & $v_{23}$ & $G_{12}$ \\
\cline { 2 - 6 } & $10.95 \pm 1.09$ & $3.55 \pm 0.32$ & $0.47 \pm 0.04$ & $0.49 \pm 0.05$ & 1.53 \\
\hline \multirow{2}{*}{ PEGDA-ISF } & \multicolumn{3}{c}{$E(\mathrm{MPa})$} & \multicolumn{2}{c}{$v$} \\
& & $4.54 \pm 0.43$ & & $0.39 \pm 0.03$ \\
\hline
\end{tabular}

namic functions [7]. However, the influence of such key factor, anisotropy, on mechanical behavior and hemodynamic property of newly developed valve prosthesis, especially PHVs, has been poorly understood. Herein, an anisotropic PHV substitute, as well as an isotropic PHV for comparison were fabricated. The effects of anisotropy on mechanical properties and hydrodynamic performance of these two PHVs have been investigated by both experimental and computational analysis.

SFs, natural polymer proteins extracted from silk, have been widely applied to artificial scaffolds and tissue engineering, such as soft tissue repair [31], bone regeneration [32] and drug delivery [33], owing to their good biocompatibility, mechanical stability and tunable properties [34]. Electrospun nanofibrous materials offer pre- cise porosity and architecture, which have shown mechanical properties, such as elastic modulus and tensile strength, similar to some native tissues $[17,35,36]$. SF can be electrospun as directional continuous fibers that provide alternative micro- to nano-scale topography [37]. Both ISF and ASF membranes were prepared by the electrospinning method for further fabrication of PHVs. The diameters of electrospun SF fibers (Fig. 2a, b) were $311.1 \pm 15.4 \mathrm{~nm}$ [38], comparable to nanofibers of leaflets (collagen fibers $\sim 70-90 \mathrm{~nm}$ in the aortic heart valve) $[39,40]$. However, SF mats alone showed low resistance to platelet adhesion and enzyme degradation in vivo $[18,41]$. PEGDA hydrogels that had shown good biocompatibility and low-fouling properties [16] were then used to prepare PEGDA-SF composites. The interpenetrating structures of PEGDA-ISF and PEGDA-ASF composites (Fig. S3a, b) were fabricated in order to enhance the integration between PEGDA hydrogels and SF fiber layers during cardiac cycles, mimicking the interpenetrating structures between different layers in heart valve leaflets as much as possible [42].

One key requirement for valve prosthesis is to provide suitable mechanical support. Anisotropic PEGDA-ASF 
PEGDA-ISF model

a Contour plots

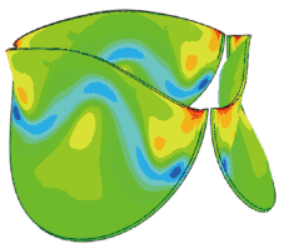

Vector plots

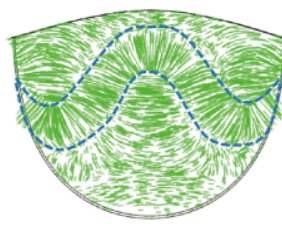

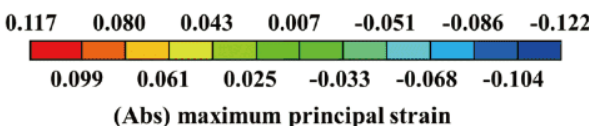

PEGDA-ASF model

b Contour plots Vector plots

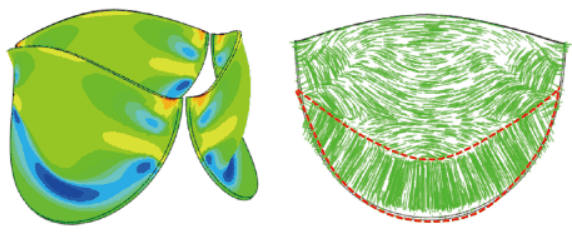

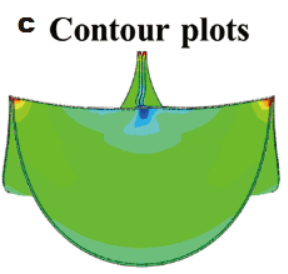

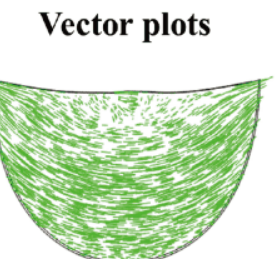

d Contour plots

\section{Vector plots}

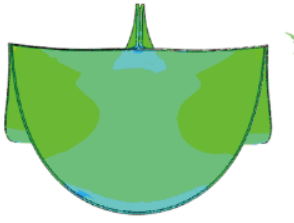

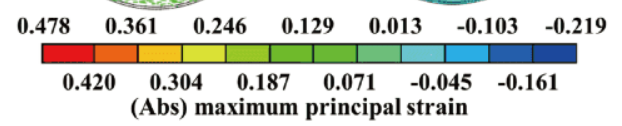

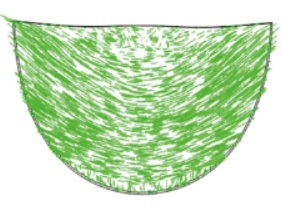

Figure 7 Contour plots and vector plots of the maximum principal strain for (a) PEGDA-ISF and (b) PEGDA-ASF leaflets in the fully open state, (c) PEGDA-ISF model and (d) PEGDA-ASF leaflets in the fully closed state.

PEGDA-ISF model

a

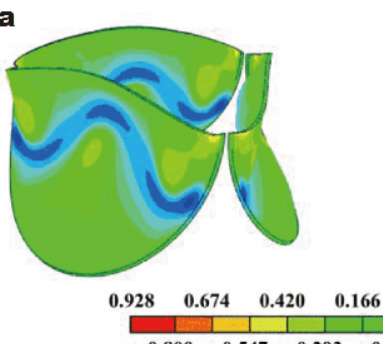

$\begin{array}{llllll}0.800 & 0.547 & 0.293 & 0.039 & -0.216 & -0.470\end{array}$

(Abs) maximum principal stress (MPa)

c

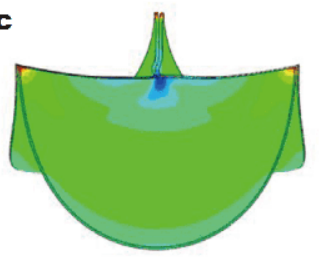

\begin{tabular}{|c|c|c|c|c|c|c|c|}
2.372 & 1.806 & 1.240 & 0.673 & 0.107 & -0.459 & -1.025 \\
\hline 2.089 & 1.523 & 0.957 & 0.390 & -0.176 & -0.742
\end{tabular}

(Abs) maximum principal stress (MPa) d

b
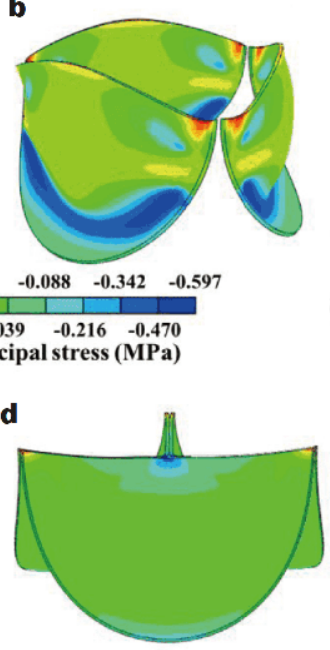

\section{.}

Figure 8 The maximum principal stress contour plots for the PEGDA-ISF valves (a, c) and PEGDA-ASF valves (b, d) in the fully open (a, b) and closed (c, d) state. (e) Frequency count distribution of relative principal stress (positive) for PEGDA-ISF valve and PEGDA-ASF valve leaflets in the fully open configuration. 

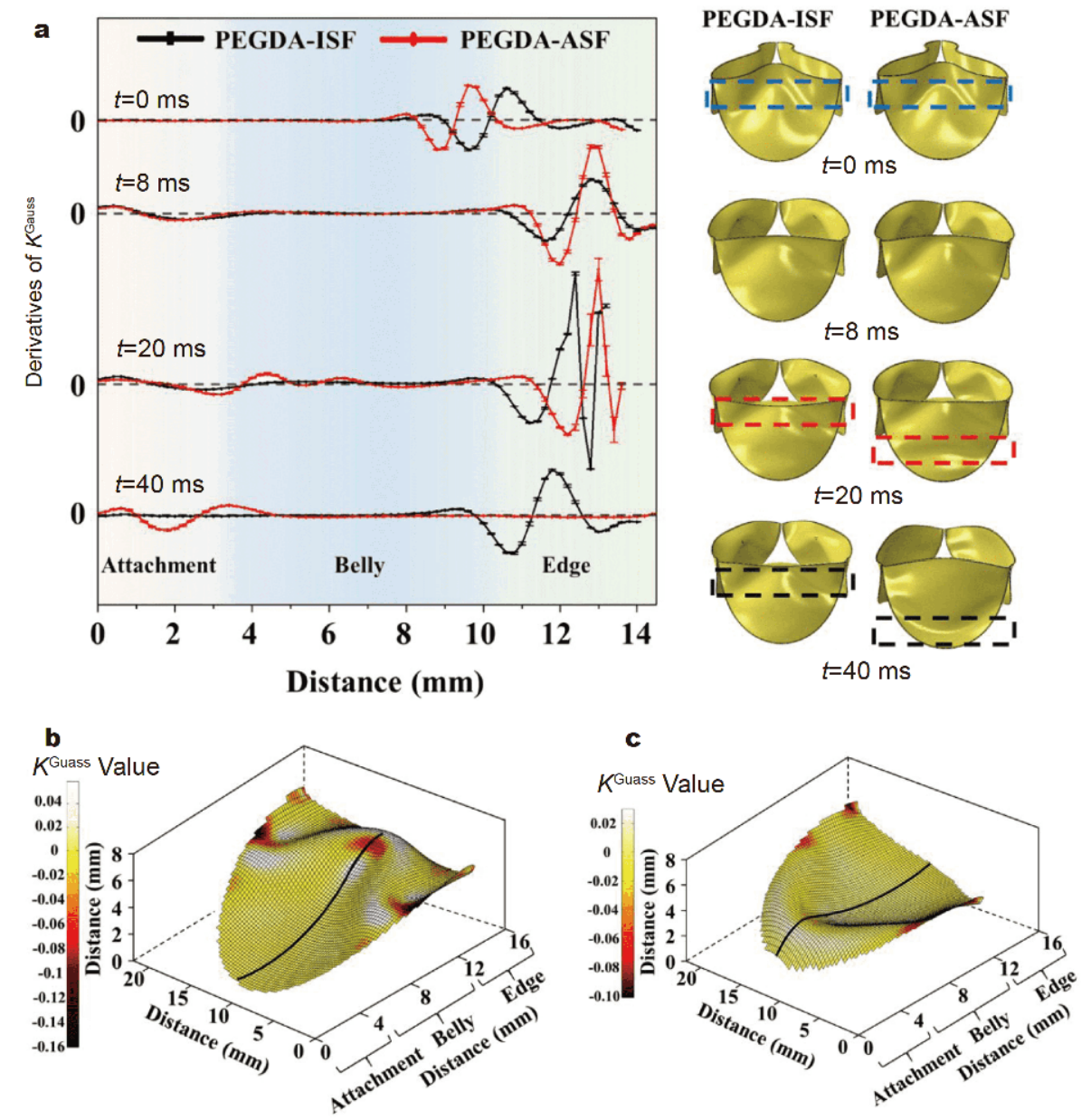

Figure 9 (a) The variation rates of Gauss curvature along the black lines of (b, c) and corresponding morphologies at different time periods during the opening process. The Gauss curvature distribution for leaflets of (b) PEGDA-ISF and (c) PEGDA-ASF in the fully open configuration ( $t=40$ ms). The black lines represent the central positions of the leaflets along the radial direction.

composites showed elastic moduli of $10.95 \pm 1.09$ and $3.55 \pm 0.32 \mathrm{MPa}$ in the directions of $\theta=0^{\circ}$ and $\theta=90^{\circ}$, respectively, close to those of native heart valves $(\sim 3-15 \mathrm{MPa}$ in the circumferential direction and $\sim 1-2 \mathrm{MPa}$ in the radial direction) [25-27]. On the other hand, the isotropic PEGDA-ISF composites presented elastic moduli of $4.54 \pm 0.43 \mathrm{MPa}$ (Fig. 4d). Another key requirement for valve prosthesis is appropriate hemodynamic property during the cardiac cycles. EOA and RF values for PHVs from both PEGDA-ASF and PEGDAISF composites can meet the requirement of ISO 5840-3 standard. Thus, PHVs from both PEGDA-ASF and PEGDA-ISF composites can be used as anisotropic and isotropic valve prostheses.

To reveal the effects of anisotropy on the mechanical behavior and hemodynamic performance of these PHVs, constitutive models were established. A number of material constitutive models have been previously developed for soft biological tissues including linear elastic models [43-45], Fung-elastic 2D/3D models [46-49] and strain invariant-based fiber-reinforced hyperelastic model $[50,51]$. In this study, isotropic linear elastic model and transversely isotropic linear elastic models were used as the constitutive models for PEGDA-ISF and PEGDAASF, respectively. Considering the orthogonal distribution of fibers in the matrix of PEGDA-ASF, five parameters, three moduli and two Poisson's ratios, were used in the constitutive model. Sample images were recorded using a CCD camera with $1600 \times 1200$ pixel resolution during the uniaxial tensile test, and strains of two orthogonal directions were analyzed to calculate Poisson's ratio and shear modulus as previously reported [52-54]. 
Cross valve pressure needs to be applied on the valve surface as the loading condition for FEA. A static uniform pressure field onto leaflets (typically $4-7 \mathrm{mmHg}$ for the open state and $120 \mathrm{mmHg}$ for the close state) was mainly used for quasistatic simulation or a linear pressure varying with time was proposed to approach the physiological transvalvular condition [55-57]. These approaches were previously adopted to acquire the stress and strain distribution; however, valve free edge may be overly expanded because of the contrived pressure when the valve was fully open [2]. To solve these problems, real hemodynamic pressure loadings on PHVs were tested using a pulse duplicator system under a normal physiological condition according to the ISO 5840-3 Standard (Fig. 5b). These material parameters and hemodynamic loading conditions for the PHVs obtained from experimental tests were further applied to FEA.

FE simulations were employed to investigate effects of anisotropy on the mechanical behavior and morphology change of PHVs from PEGDA-ISF and PEGDA-ASF composites under cardiac cycles. Excessive deformation areas with large maximum principal strain were found for both PEGDA-ISF and PEGDA-ASF leaflets during systole, which considerably affected the open configuration (Fig. 7a, b). The bending area of the PEGDA-ASF leaflet was closer to the attachment line with a smooth free edge. However, the bending area for the PEGDA-ISF leaflet remained near the free edge when fully open, resulting in abnormal surface morphology and large stress concentration (Fig. 8e). Gauss curvature changes (Fig. 9b, c) at the fully open state also confirmed that surface morphology for the PEGDA-ISF leaflet was much rougher than that for the PEGDA-ASF leaflet. Difference in morphology change during systole may be attributed to isotropy and anisotropy of these PHVs. Compared with the isotropic PEGDA-ISF leaflet, the anisotropic PEGDAASF leaflet was more flexible in the radial direction, resulting in appropriate morphology change during systole similar to biological heart valve (e.g., bovine pericardial valve, porcine heart valve) as previously reported $[53,54,58,59]$. The hydrodynamic curves of the PHVs from PEGDA-ASF composites (Fig. 5b) exhibited a lower mean pressure difference $(1.43 \mathrm{mmHg}$, green shadow areas) than that of PHVs from PEGDA-ISF composites (3.79 mmHg, Fig. $5 c$, Table S1). Comparing with PEGDA-ISF samples, PEGDA-ASF samples are more flexible in the radial direction similar to native heart valves, resulting in fast fully opening of leaflets and smooth blood flow in systole (larger EOA value and lower energy loss). These results confirm that anisotropy of PHVs influences their hemodynamic properties.

Aortic valve leaflets bear peak differential pressures about $100 \mathrm{mmHg}$ under normotensive conditions during diastole [6]. Mechanical anisotropy enables the leaflets to restrict the circumferential deformation while simultaneously enlarging in the radial direction to achieve proper closure. Our results showed the maximum principal stress predominantly along the circumferential direction in the fully closed state (Fig. 7c, d), parallel to the fiber alignment direction of the PEGDA-ASF leaflet. The fibers in the PEGDA-ASF leaflets aligned along the circumferential direction can provide sufficient strength and are thus resilient to peak stress during diastole. The anisotropic property of the PEGDA-ASF leaflets also resulted in a slight decrease of the peak stress value $(2.20 \mathrm{MPa})$, compared with that from the isotropic PEGDA-ISF leaflet $(2.37 \mathrm{MPa})$. A previous study also showed the decrease of peak stress from $1.42 \mathrm{MPa}$ in the isotropic bovine pericardium valve model to $1.13 \mathrm{MPa}$ in the anisotropic model through computational modeling [60]. Therefore, anisotropic PEGDA-ASF leaflets with layered fibrous structures can mimic the circumferential alignment of collagen fibers in native heart valves, as well as their response to mechanical stimuli [61], and attenuate the stress concentration under closing pressure gradient, which may improve the durability of the PHV prosthesis.

\section{CONCLUSIONS}

In this study, anisotropic and isotropic PHVs were prepared using PEGDA-ASF and PEGDA-ISF composites, respectively. The PEGDA-ASF composites showed anisotropic characteristics and mechanical properties close to those from aortic valve leaflets, while the PEGDA-ISF composites exhibited isotropic mechanical behavior. The hydrodynamic properties of PHVs from both PEGDADSF and PEGDA-ASF composites met the requirements of the ISO 5840-3 Standard using the pulse duplicator tests. However, FEA results revealed that the anisotropic PEGDA-ASF valve showed a lower maximum principal stress value in commissures during diastole compared with that from the isotropic PEGD-ISF valve. The Gauss curvature analysis indicated that the anisotropic PEGDAASF valve can produce appropriate surface morphology by dynamically adjusting the movement of bending area during the opening process. Thus, the anisotropic characteristics of PHVs played significant roles in their mechanical and hemodynamic behavior, which should be considered in the development of novel PHVs. 
Received 18 September 2019; accepted 11 November 2019; published online 12 December 2019

1 Zhao Z, Fang R, Rong Q, et al. Bioinspired nanocomposite hydrogels with highly ordered structures. Adv Mater, 2017, 29: 1703045

2 Jana S, Levengood SKL, Zhang M. Anisotropic materials for skeletal-muscle-tissue engineering. Adv Mater, 2016, 28: 10588-10612

3 Fratzl P, Weinkamer R. Nature's hierarchical materials. Prog Mater Sci, 2007, 52: 1263-1334

4 Wu S, Duan B, Qin X, et al. Living nano-micro fibrous woven fabric/hydrogel composite scaffolds for heart valve engineering. Acta Biomater, 2017, 51: 89-100

5 Sun W, Martin C, Pham T. Computational modeling of cardiac valve function and intervention. Annu Rev Biomed Eng, 2014, 16: 53-76

6 Pham T, Sulejmani F, Shin E, et al. Quantification and comparison of the mechanical properties of four human cardiac valves. Acta Biomater, 2017, 54: 345-355

7 Ghanbari H, Viatge H, Kidane AG, et al. Polymeric heart valves: New materials, emerging hopes. Trends Biotech, 2009, 27: 359-367

8 Tseng H, Puperi DS, Kim EJ, et al. Anisotropic poly(ethylene glycol)/polycaprolactone hydrogel-fiber composites for heart valve tissue engineering. Tissue Eng Part A, 2014, 20: 2634-2645

9 Masoumi N, Annabi N, Assmann A, et al. Tri-layered elastomeric scaffolds for engineering heart valve leaflets. Biomaterials, 2014, 35: 7774-7785

10 Hosseini V, Evrova O, Hoerstrup SP, et al. A simple modification method to obtain anisotropic and porous 3D microfibrillar scaffolds for surgical and biomedical applications. Small, 2018, 14: 1702650

11 Burriesci G, Howard IC, Patterson EA. Influence of anisotropy on the mechanical behaviour of bioprosthetic heart valves. J Med Eng Tech, 1999, 23: 203-215

12 Li J, Luo XY, Kuang ZB. A nonlinear anisotropic model for porcine aortic heart valves. J Biomech, 2001, 34: 1279-1289

13 Abbasi M, Barakat MS, Vahidkhah K, et al. Characterization of three-dimensional anisotropic heart valve tissue mechanical properties using inverse finite element analysis. J Mech Behav Biomed Mater, 2016, 62: 33-44

14 Koch TM, Reddy BD, Zilla P, et al. Aortic valve leaflet mechanical properties facilitate diastolic valve function. Comput Methods BioMech BioMed Eng, 2010, 13: 225-234

15 Saleeb AF, Kumar A, Thomas VS. The important roles of tissue anisotropy and tissue-to-tissue contact on the dynamical behavior of a symmetric tri-leaflet valve during multiple cardiac pressure cycles. Med Eng Phys, 2013, 35: 23-35

16 Li Q, Bai Y, Jin T, et al. Bioinspired engineering of poly(ethylene glycol) hydrogels and natural protein fibers for layered heart valve constructs. ACS Appl Mater Interfaces, 2017, 9: 16524-16535

17 Rockwood DN, Preda RC, Yücel T, et al. Materials fabrication from bombyx mori silk fibroin. Nat Protoc, 2011, 6: 1612-1631

18 Jin $\mathrm{H}$, Chen J, Karageorgiou V, et al. Human bone marrow stromal cell responses on electrospun silk fibroin mats. Biomaterials, 2004, 25: 1039-1047

19 DeLong SA, Moon JJ, West JL. Covalently immobilized gradients of BFGF on hydrogel scaffolds for directed cell migration. Biomaterials, 2005, 26: 3227-3234

20 Endo VT, de Carvalho Pereira JC. Linear orthotropic viscoelasticity model for fiber reinforced thermoplastic material based on Prony series. Mech Time-Depend Mater, 2017, 21: 199-221

21 ISO 5840-3: Cardiovascular implants-cardiac valve prosthesestranscatheter techniques. 2015

22 Wang M, Jin HJ, Kaplan DL, et al. Mechanical properties of electrospun silk fibers. Macromolecules, 2004, 37: 6856-6864

23 Tsukada M, Gotoh Y, Nagura M, et al. Structural changes of silk fibroin membranes induced by immersion in methanol aqueous solutions. J Polym Sci B Polym Phys, 1994, 32: 961-968

24 Tsou L, Sauer JA, Hara M. Molecular composites of poly( $p$ phenylene terephthalamide) anion and poly(ethylene oxide): Thermal behavior and morphology. J Polym Sci B Polym Phys, 2000, 38: 1369-1376

25 Clark RE. Stress-strain characteristics of fresh and frozen human aortic and mitral leaflets and chordae tendineae. Implications for clinical use. J Thorac Cardiovasc Surg, 1973, 66: 202-208

26 Missirlis YF, Chong M. Aortic valve mechanics-Part I: Material properties of natural porcine aortic valves. J Bioeng, 1978, 2: 287300

27 Stradins P, Lacis R, Ozolanta I, et al. Comparison of biomechanical and structural properties between human aortic and pulmonary valve. Eur J Cardiothorac Surg, 2004, 26: 634-9

28 Yoganathan AP, He Z, Casey Jones S. Fluid mechanics of heart valves. Annu Rev Biomed Eng, 2004, 6: 331-362

29 Rees DWA. Basic Engineering Plasticity: An Introduction with Engineering and Manufacturing Applications. Amsterdam: Elsevier, 2006. 23-42

30 Sritharan D, Fathi P, Weaver JD, et al. Impact of clinically relevant elliptical deformations on the damage patterns of sagging and stretched leaflets in a bioprosthetic heart valve. Cardiovasc Eng Tech, 2018, 9: 351-364

31 Sang Y, Li M, Liu J, et al. Biomimetic silk scaffolds with an amorphous structure for soft tissue engineering. ACS Appl Mater Interfaces, 2018, 10: 9290-9300

32 Naskar D, Bhattacharjee P, Ghosh AK, et al. Carbon nanofiber reinforced nonmulberry silk protein fibroin nanobiocomposite for tissue engineering applications. ACS Appl Mater Interfaces, 2017, 9: $19356-19370$

33 Hassani Besheli N, Mottaghitalab F, Eslami M, et al. Sustainable release of vancomycin from silk fibroin nanoparticles for treating severe bone infection in rat tibia osteomyelitis model. ACS Appl Mater Interfaces, 2017, 9: 5128-5138

34 Ma L, Akurugu MA, Andoh V, et al. Intrinsically reinforced silks obtained by incorporation of graphene quantum dots into silkworms. Sci China Mater, 2019, 62: 245-255

35 Gong W, Lei D, Li S, et al. Hybrid small-diameter vascular grafts: Anti-expansion effect of electrospun poly $\varepsilon$-caprolactone on heparin-coated decellularized matrices. Biomaterials, 2016, 76: 359370

36 Yao D, Liu H, Fan Y. Fabrication of water-stable silk fibroin scaffolds through self-assembly of proteins. RSC Adv, 2016, 6: 61402-61409

37 Chen S, Lou Z, Chen D, et al. Highly flexible strain sensor based on $\mathrm{ZnO}$ nanowires and $\mathrm{P}(\mathrm{VDF}-\mathrm{TrFE})$ fibers for wearable electronic device. Sci China Mater, 2016, 59: 173-181

38 Ghosh S, Laha M, Mondal S, et al. In vitro model of mesenchymal condensation during chondrogenic development. Biomaterials, 2009, 30: 6530-6540

39 Jastrzebska M, Zalewska-Rejdak J, Mróz I, et al. Atomic force microscopy and FT-IR spectroscopy investigations of human heart valves. Gen Physiol Biophys, 2006, 25: 231-244 
Zhang F, Zuo B, Fan Z, et al. Mechanisms and control of silk-based electrospinning. Biomacromolecules, 2012, 13: 798-804

41 Rodriguez MJ, Brown J, Giordano J, et al. Silk based bioinks for soft tissue reconstruction using 3-dimensional (3D) printing with in vitro and in vivo assessments. Biomaterials, 2017, 117: 105-115

42 Tseng $\mathrm{H}$, Grande-Allen KJ. Elastic fibers in the aortic valve spongiosa: A fresh perspective on its structure and role in overall tissue function. Acta Biomater, 2011, 7: 2101-2108

43 Wenk JF, Zhang Z, Cheng G, et al. First finite element model of the left ventricle with mitral valve: Insights into ischemic mitral regurgitation. Ann Thoracic Surgery, 2010, 89: 1546-1553

$44 \mathrm{Xu} \mathrm{C}$, Brinster CJ, Jassar AS, et al. A novel approach to in vivo mitral valve stress analysis. Am J Physiol-Heart Circulat Physiol, 2010, 299: H1790-H1794

45 Krishnamurthy G, Ennis DB, Itoh A, et al. Material properties of the ovine mitral valve anterior leaflet in vivo from inverse finite element analysis. Am J Physiol-Heart Circulatory Physiol, 2008, 295: H1141-H1149

46 Fung YC. Biomechanics: Mechanical Properties of Living Tissues. New York: Springer-Verlag, 1981. 242-314

47 Humphrey JD. Cardiovascular Solid Mechanics: Cells, Tissues and Organs. New York: Springer-Verlag, 2002. 601-712

48 Labrosse MR, Boodhwani M, Sohmer B, et al. Modeling leaflet correction techniques in aortic valve repair: A finite element study. J BioMech, 2011, 44: 2292-2298

49 Labrosse MR, Lobo K, Beller CJ. Structural analysis of the natural aortic valve in dynamics: From unpressurized to physiologically loaded. J BioMech, 2010, 43: 1916-1922

50 Holzapfel GA, Gasser TC, Ogden RW. A new constitutive framework for arterial wall mechanics and a comparative study of material models. J Elasticity, 2000, 61: 1-48

51 Weiss JA, Maker BN, Govindjee S. Finite element implementation of incompressible, transversely isotropic hyperelasticity. Comput Methods Appl Mech Eng, 1996, 135: 107-128

52 Weiler M, Hwai Yap C, Balachandran K, et al. Regional analysis of dynamic deformation characteristics of native aortic valve leaflets. J BioMech, 2011, 44: 1459-1465

53 Avelar AHF, Canestri JA, Bim C, et al. Quantification and analysis of leaflet flutter on biological prosthetic cardiac valves. Artificial Organs, 2017, 41: 835-844

54 Leo HL, Dasi LP, Carberry J, et al. Fluid dynamic assessment of three polymeric heart valves using particle image velocimetry. Ann Biomed Eng, 2006, 34: 936-952

55 Sun W, Abad A, Sacks MS. Simulated bioprosthetic heart valve deformation under quasi-static loading. J BioMech Eng, 2005, 127: 905-914

56 Avanzini A, Battini D. Structural analysis of a stented pericardial heart valve with leaflets mounted externally. Proc Inst Mech Eng H, 2014, 228: 985-995

57 Auricchio F, Conti M, Ferrara A, et al. Patient-specific simulation of a stentless aortic valve implant: The impact of fibres on leaflet performance. Comput Methods Biomech Biomed Eng, 2014, 17: 277-285

58 Yap CH, Kim HS, Balachandran K, et al. Dynamic deformation characteristics of porcine aortic valve leaflet under normal and hypertensive conditions. Am J Physiol-Heart Circulatory Physiol, 2010, 298: H395-H405

59 Sugimoto H, Sacks MS. Effects of leaflet stiffness on in vitro dynamic bioprosthetic heart valve leaflet shape. Cardiovasc Eng Tech, 2013, 4: 2-15
60 Mao W, Li K, Sun W. Fluid-structure interaction study of transcatheter aortic valve dynamics using smoothed particle hydrodynamics. Cardiovasc Eng Tech, 2016, 7: 374-388

61 Ristori T, Bouten CVC, Baaijens FPT, et al. Predicting and understanding collagen remodeling in human native heart valves during early development. Acta Biomater, 2018, 80: 203-216

Acknowledgements This work was supported by the National Natural Science Foundation of China (31300788), and the Hundred-Talent Program from Chinese Academy of Sciences. We thank Prof. Dongmei Cun and Prof. Xin Che at Shenyang Pharmaceutical University for assistance with electrospinning experiments. We also thank Wei Cui at the Institute of Metal Research, Chinese Academy of Sciences for the help with SEM analysis.

Author contributions Zhang X and Niu D conceived and supervised the project. Guo F, Liu C, Han R and Lu Q designed and carried out the experiments. Guo F, Liu C and Bai Y analyzed the data and prepared the manuscript. Yang $\mathrm{R}$ and Zhang $\mathrm{X}$ revised the manuscript. Zhang $\mathrm{X}$ and Niu D are co-corresponding authors of this paper. All authors participated in the discussion of this research.

Conflict of interest The authors declare that they have no conflict of interest.

Supplementary information

Supporting data are available in the online version of the paper.

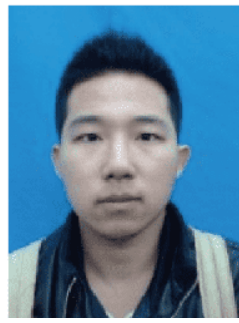

Feng Guo received his bachelor degree from the College of Sciences, Northeastern University in 2016. He is currently a PhD candidate at the Institute of Metal Research, Chinese Academy of Sciences. His research focuses on the advanced polymer artificial heart valve material and finite element simulation for fluid-structure interaction.

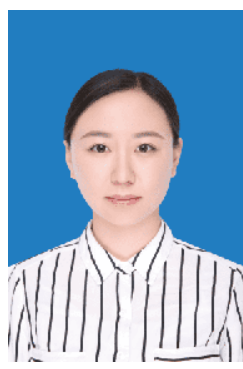

Chang Liu received her master's degree in chemical engineering from Northeastern University in 2018. She is currently a PhD student at The Hong Kong Polytechnic University. Her research interests focus on photo-crosslinking of silk fibroin and advanced fibrous filters.

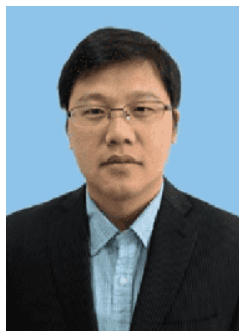

Xing Zhang received his $\mathrm{PhD}$ degree in materials science and engineering from the University of California, San Diego in 2007. He is currently a professor in materials science at the Institute of Metal Research, Chinese Academy of Sciences. His research focuses on polymeric heart valves and biofunctional bone substitutes. 
仿生各向异性高分子人工瓣膜制备及性能研究

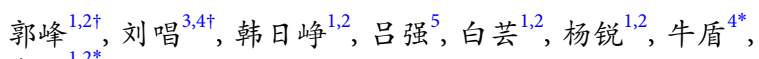
张兴 $1,2^{2}$

摘要 人体心脏瓣膜具有力学各向异性特征, 使其能够承受长期的 开合循环负荷. 本文采用静电纺丝法分别制备了具有各向异性(anisotropic silk fibroin, ASF)和各向同性(isotropic silk fibroin, ISF)的 丝素蛋白纤维膜, 并进一步与聚乙二醇二丙烯酸酯(poly(ethylene glycol) diacrylate, PEGDA)水凝胶结合, 作为人工高分子瓣膜材料 (PEGDA-ASF和PEGDA-ISF). 有限元分析结果表明PEGDA-ASF瓣 膜在心脏舒张期的最大主应力值 $(2.20 \mathrm{MPa}$ ) 低于PEGDA-ISF瓣膜 $(2.37 \mathrm{MPa})$. 与人体瓣膜相似, 收缩期时PEGDA-ASF瓣膜在瓣叶根 部附近会产生一个弯折区域, 而PEGDA-ISF瓣膜的弯折区却接近 瓣叶的自由边缘. 此外, PEGDA-ASF瓣膜在打开过程中, 能够通过 动态调整弯折区域获得较为平滑的表面形貌. 因此, 人工高分子瓣 膜的各向异性特征对于实现其与人体瓣膜相似的力学和流体动力 学行为起着至关重要的作用. 